\title{
Clinical utility versus futility: a tipping point for liquid biopsies in bladder cancer
}

\author{
Erik Kouba ${ }^{1}$ \& Liang Cheng *,2,3 (iD \\ ${ }^{1}$ Associated Pathologists at Medical Center of Central Georgia, Macon, GA 46202, USA \\ ${ }^{2}$ Department of Pathology \& Laboratory Medicine, Indiana University School of Medicine, Indianapolis, IN 46202, USA \\ ${ }^{3}$ Department of Urology, Indiana University School of Medicine, Indianapolis, IN 46202, USA \\ *Author for correspondence: Tel.: +1 317491 6442; Fax: +1 317491 6419; liang_cheng@yahoo.com
}

\begin{abstract}
"Liquid biopsy based biomarker may find use in risk stratification, improvement in recurrence metastatic detection, assessment of treatment response and prediction of treatment efficacy"
\end{abstract}

First draft submitted: 28 August 2019; Accepted for publication: 9 September 2019; Published online: 25 October 2019

Keywords: biomarker $\bullet$ cell-free DNA • liquid biopsy • precision medicine $\bullet$ urinary bladder

\section{Highlights}

With the emergence of genomic profiling and the drive toward targeted molecular therapies, cancer-specific biomarkers have undergone rapid concurrent development for use in precision-based medicine. From a basic standpoint, such biomarkers represent objectively measured biomolecules such as DNA, RNA, protein or peptide modifications within normal or abnormal biological states. Novel biotechnologies have shed insight into hitherto unknown genomic and proteomic alterations and potential techniques for their detection, isolation and interpretation. In addition, there has been an expansion of the fundamental genetic understanding of perturbed biological states within clinical oncology. These critical molecular and cellular biomarkers of such aberrations represent the hallmark of cancer and formulate the foundation of the multitude of studies aimed at change in clinical practice [1-3].

Characterization of tumor-derived cell-free DNA (ctDNA) found in blood samples is termed liquid biopsy and represents a significant technological advancement with potential clinical applications [4-7]. To date, decisions for management of urothelial carcinoma of the urinary bladder (UCB) is based upon traditional pathological characteristic features of the primary tumor radiological findings for initial staging and detection of subsequent metastases and/or recurrence which is suboptimal and fraught with inherent limitations [8-10]. As molecular characterization of tumors has become increasingly important toward understanding factors impacting the clinical management of $\mathrm{UCB}, \mathrm{ctDNA}$ has become increasingly attractive as a biomarker with potential means to facilitate the diagnosis, treatment selection, monitoring treatment response, tracking minimal disease and treatment resistance [11].

The 'tipping point' is a term used to describe the moment in time in which incremental increases in technological understanding culminates in an expansion of knowledge which creates change. For molecular diagnostic testing, this 'tipping point' represents the transformation of fundamental genetic discovery into application and subsequent utilization for use in the improved clinical care of patients [12]. Despite the emergence of advances in technologies has ctDNA reached such a 'tipping point' therefore enabling trials for improved outcome directed management of UCB? In other words, has clinical utility been demonstrated? If so, such a validated biomarker is ready for implementation including regulatory approval, commercialization and incorporation into clinical management guidelines [13]. In this editorial, we examine the current state of liquid biopsy in the use of clinical management of UCB from the overview of a recent clinical study published in the May 2019 issue of Journal of Clinical Oncology. Conclusions regarding this 'tipping point' in terms of current clinical utility of such assays may be obtained from the results.

Earlier studies using liquid biopsy specimens and ctDNA have shown the possibility to identify early metastatic disease detection, disease progression and detect actionable genetic targets in patients with UCB. Most of these studies were proof-of-principle with limited numbers of patients incorporating several types of genetic testing platforms [14-18]. However, more recently Christensen et al. have published the results of a comprehensive single-

Future $\because$ Medicine 
center, prospective study of 68 patients with clinically localized bladder cancer and longitudinal analysis of ctDNA during well-defined stages of UCB treatment and progression. The study used a personalized tissue-based blood test to track ctDNA at a priori defined individual disease timepoints during conventional management of UCB [19].

In this study, all the patients underwent diagnostic transurethral resection for tissue diagnosis at the same time undergoing standard PET/CT staging [19]. Concurrent with this time point blood samples were obtained and DNA was extracted according to a standardized procedure. Subsequently, exome sequencing was performed using libraries of tumor and matching germline DNA to identify mutational signatures previously identified in bladder cancer. Patient-specific somatic variants were selected using plasma multiplex NGS sequencing and matched with clonal mutations after DNA extraction from plasma samples (Signatera ${ }^{T M}$, Natera, Inc.). Sample-level analytic sensitivity has previously been determined to be greater than $95 \%$ at a $0.01 \% \mathrm{ctDNA}$ concentration level.

After initial staging, subjects underwent neoadjuvant chemotherapy using gemcitabine/cisplatin. After appropriate treatment, all patients underwent radical cystectomy with extended lymphadenectomy to the level of the aortic bifurcation (either by open- or robotic-assisted approach). Patients were subsequently followed using CT imaging of thorax and abdomen at regularly scheduled visits. During these visits, plasma specimens were procured from the subjects and genetic expression of ctDNA was analyzed by the aforementioned platform.

The analysis of the detected ctDNA of the subjects over the study period (2013-2017) was shown to have potential utility in terms of clinical meaningful prognosis and disease progression of individuals at several time points of interest. The presence of ctDNA after chemotherapy was associated with pathologic outcomes after subsequent cystectomy. Quite remarkably, 100\% of patients with precystectomy detectable ctDNA had residual tumor and/or lymph node metastatses. On the contrary, those with ypT0 pathology were ctDNA negative. After $\mathrm{NAC}$ and cystectomy, there also was a significant difference in overall recurrence rates between patients with detectable ctDNA and those without (75 vs $0 \%$, respectively, $\mathrm{p}<0.001) .12$-month recurrence rates also showed a similar relationship between the two groups. Moreover, in multivariate Cox proportional hazard regression, ctDNA status after cystectomy was the strongest predictor of recurrence-free survival. The use of longitudinal detection of ctDNA following cystectomy was shown to have a significant lead time over conventional imaging for the detection of clinical recurrence (mean, 96 days). When using strict criteria, serial analysis identified metastatic relapse with $100 \%$ sensitivity and $98 \%$ specificity. Overall, the results suggest that the presence of ctDNA identifies patients with high-risk disease progression. This biomarker was independent of RNA subtype (basal/squamous, luminal papillary, luminal unstable, stroma-rich), mutational burden and selected gene sets related to chemotherapy response (e.g., ECCR2 mutations, $D D R$ mutations, and immune related gene sets). Moreover, when comparing whole exome sequencing of primary tissue specimens and ctDNA, the investigators found a high degree of similarity in the mutational landscapes between the two suggesting a limited clonal evolution in the disease progression of these selected subjects [19].

While an increasing number of studies show the potential use of ctDNA as a biomarker in applications of cancer management, the feasibility of such management requires foremost the optimization and standardization of preanalytical and analytical methodology. It is within this realm that current gaps in the knowledge may hinder translation to clinical practice [20]. The study by Christensen $e$ t al. addresses the issues associated with robust sample processing, technical soundness of assays, reproducibility of bioinformatic analysis, the technological and analytical limitations associated with detecting low mutant allele fractions in serum of patients with UCB. In the current study, quality control performed during the workflow showed a 99\% sample integrity rate (651 of 656 plasma specimens). While not US FDA approved, the ctDNA platform employed in the study has received 'Breakthrough Device Designation' which offers increased interaction in terms of future regulatory submissions (www.FDA.gov).

Overall, the results of the study suggest that ctDNA may allow guidance in the treatment of patients with UCB. Liquid biopsy based biomarker may find use in risk stratification, improvement in recurrence metastatic detection, assessment of treatment response and prediction of treatment efficacy. Current investigations indicate that longitudinal ctDNA response provides patient-level insight into therapeutic intervention in longitudinal analysis of liquid biopsies of various tumor types, including breast cancer, non-small-cell lung cancer and colorectal cancer [11,21-23]. For non-small-cell lung cancer, ctDNA is used to guide clinical management using EGFR mutational analysis. Studies are also underway evaluating the potential assessment of genetic alterations in liquid biopsy for early detection of various cancer types [7,24,25].

What may we conclude regarding the current state in UCB if we reflect on the various stages of cancer biomarker development in the context of developmental stages in other malignancies? In view of the study by Christensen et al., we observe that ctDNA has been defined as a predictive and outcome-directed biomarker with specimen 
stability, assay reproducibility and availability. Based on the data discussed herein, it appears that the critical 'tipping point' has been met in the development of ctDNA as a potential biomarker in UCB. The next challenges in terms of improving patient outcomes will require investigation in prospective interventional studies to demonstrate significant advantages over existing technology. Meeting such criterion will foster clinical implementation and incorporation into practice guidelines.

\section{Financial \& competing interests disclosure}

The authors have no relevant affiliations or financial involvement with any organization or entity with a financial interest in or financial conflict with the subject matter or materials discussed in the manuscript. This includes employment, consultancies, honoraria, stock ownership or options, expert testimony, grants or patents received or pending, or royalties.

No writing assistance was utilized in the production of this manuscript.

\section{References}

1. Cheng L, Eble JN. Molecular Surgical Pathology. Springer, NY, USA (2013).

2. Cheng L, Zhang DY, Eble JN. Molecular Genetic Pathology. Springer, NY, USA (2013).

3. Bardelli A, Pantel K. Liquid biopsies, what we do not know (yet). Cancer Cell 31, 172-179 (2017).

4. Saarenheimo J, Eigeliene N, Andersen $\mathrm{H}$ et al. The value of liquid biopsies for guiding therapy decisions in non-small cell lung cancer. Front. Oncol. 9, 129 (2019).

5. Riethdorf S, Soave A, Rink M. The current status and clinical value of circulating tumor cells and circulating cell-free tumor DNA in bladder cancer. Transl. Androl. Urol. 6, 1090-1110 (2017).

6. Otandault A, Anker P, Al Amir Dache Z et al. Recent advances in circulating nucleic acids in oncology. Ann. Oncol. 30, 374-384 (2019).

7. Pantel K, Alix-Panabieres C. Liquid biopsy and minimal residual disease - latest advances and implications for cure. Nat. Rev. Clin. Oncol. 16, 409-424 (2019).

8. Cheng L, Lopez-Beltran A, Bostwick DG. Bladder Pathology. Wiley-Blackwell, NJ, USA (2012).

9. Cheng L, MacLennan GT, Bostwick DG. Urologic Surgical Pathology. Elsevier, PA, USA (2019).

10. Cheng L, Zhang S, MacLennan GT et al. Bladder cancer: translating molecular genetic insights into clinical practice. Hum. Pathol. 42, 455-481 (2011).

11. Rossi G, Ignatiadis M. Promises and pitfalls of using liquid biopsy for precision medicine. Cancer Res.79, 2798-2804 (2019).

12. Adams KT. Gene-based diagnostics: ready for prime time? Biotechnol. Healthc. 2, 20-28 (2005).

13. Goossens N, Nakagawa S, Sun X, Hoshida Y. Cancer biomarker discovery and validation. Transl. Cancer Res. 4, 256-269 (2015).

14. Vandekerkhove G, Todenhofer T, Annala M et al. Circulating tumor DNA reveals clinically actionable somatic genome of metastatic bladder cancer. Clin. Cancer Res. 23, 6487-6497 (2017).

15. Agarwal N, Pal SK, Hahn AW et al. Characterization of metastatic urothelial carcinoma via comprehensive genomic profiling of circulating tumor DNA. Cancer 124, 2115-2124 (2018).

16. Christensen E, Birkenkamp-Demtroder K, Nordentoft I et al. Liquid biopsy analysis of FGFR3 and PIK3CA hotspot mutations for disease surveillance in bladder cancer. Eur. Urol. 71, 961-969 (2017).

17. Birkenkamp-Demtroder K, Christensen E, Nordentoft I et al. Monitoring treatment response and metastatic relapse in advanced bladder cancer by liquid biopsy analysis. Eur. Urol. 73, 535-540 (2018).

18. Todenhofer T, Struss WJ, Seiler R et al. Liquid biopsy-analysis of circulating tumor DNA (ctDNA) in bladder cancer. Bladder Cancer 4, 19-29 (2018).

19. Christensen E, Birkenkamp-Demtroder K, Sethi H et al. Early detection of metastatic relapse and monitoring of therapeutic efficacy by ultra-deep sequencing of plasma cell-free DNA in patients with urothelial bladder carcinoma. J. Clin. Oncol. 37, 1547-1557 (2019).

20. Bronkhorst AJ, Ungerer V, Holdenrieder S. The emerging role of cell-free DNA as a molecular marker for cancer management. Biomol. Detect. Quantif. 17, 100087 (2019).

21. Abbosh C, Birkbak NJ, Swanton C. Early stage NSCLC - challenges to implementing ctDNA-based screening and MRD detection. Nat. Rev. Clin. Oncol. 15, 577-586 (2018).

22. Reinert T, Henriksen TV, Christensen E et al. Analysis of plasma cell-free DNA by ultradeep sequencing in patients with stages I to III colorectal cancer. JAMA Oncol. (2019) (in press).

23. Buono G, Gerratana L, Bulfoni M et al. Circulating tumor DNA analysis in breast cancer: is it ready for prime-time? Cancer Treat. Rev. 73, 73-83 (2019).

24. Dudley JC, Schroers-Martin J, Lazzareschi DV et al. Detection and surveillance of bladder cancer using urine tumor DNA. Cancer Discov. 9, 500-509 (2019).

25. Kaiser J. 'Liquid biopsy’ for cancer promises early detection. Science 359, 259 (2018). 
